\title{
A Review of IFM-Free and IFM-Based Methods For Underwater Imaging
}

\author{
Henna Francis ${ }^{1}$, Anjusree V K ${ }^{2}$ \\ ${ }^{1}$ Student, Department of Computer Science and Information System, Rajagiri School Of Engineering And \\ Technology,Ernakulam, India, hennafrancis97@gmail.com \\ ${ }^{2}$ Assistant Professor, Department of Computer Science and Engineering, Rajagiri School Of Engineering And \\ Technology,Ernakulam, India, anjusreevk@ rajagiritech.edu.in
}

\begin{abstract}
Underwater images play a key role in ocean exploration, however typically suffer from severe quality degradation because of light absorption and scattering in water medium. Researchers attempt to capture high-quality underwater pictures for a range of underwater applications. The particles suspended underwater generate unwanted noise and worsens the visibility of dimming pictures. The specialised hardware platforms and cameras may be pricey and power-consuming. Several algorithms are developed for underwater image quality improvement by image enhancement or restoration. To review current development of quality improvement methods for underwater images comprehensive comparisons on IFMBased underwater image restoration methods and IFMFree underwater image enhancement methods from both subjective and objective perspectives are used in this paper.. This paper introduces the essential principles of underwater imaging models with image enhancement and image restoration.
\end{abstract}

Key words: IFM-Free, IFM-Based, MIP, DCP,CLAHE, RAYLEIGH.

\section{INTRODUCTION}

Underwater images play a key role in ocean exploration. Researchers attempt to capture high-quality underwater pictures for a range of underwater applications, together with artificial intelligence and rescue missions. The quality of underwater pictures is severely littered with the actual physical and chemical characteristics of underwater conditions. The particles that are suspended underwater absorb bulk of light energy. This will modify the direction of light before the light mirrored from the underwater scene reaches the camera and results in pictures having low-contrast, blur and haze. So as to extend the range of underwater imaging, artificial light-weight sources are typically used. Images are degraded by the influence of water cloudiness, light-weight absorption and scattering. Extracting valuable information for underwater scenes needs effective ways to correct color, improve clarity, address blurring and background scattering. The applicability of the latest ways for underwater images are extremely high. Many algorithms are developed for underwater image quality improvement by image enhancement or restoration. Reviewed a lot of aspects of the underwater optical process, together with underwater image de-scattering, underwater image restoration and underwater image quality assessments. This paper provides a broader review, an experimental-based comparison of key methods and provides a clear idea of each method through comparisons. The scope of the work is to take the experimental based review of image enhancement and image restoration ways for underwater imaging. Objective of this work is to check every algorithm of IFM-Free and IFM-Based ways, to induce the clear plan of underwater image enhancement and restoration techniques. Extracting valuable information for underwater scenes needs effective ways to correct color, improve clarity, address blurring and background scattering. These are particularly challenging because of the complicated underwater setting, wherever images are degraded by the influence of water cloudiness, light-weight absorption and scattering, which can be modified generally. Understanding the underwater optical imaging model could help us better design and propose robust and effective enhancement strategies.

\section{METHODS}

Underwater images are having main consideration in ocean exploration, however usually suffer from severe quality degradation due to light-weight absorption and scattering in water medium. Though major breakthroughs are created recently within the general space of image enhancement and restoration, the applicability of latest strategies for raising the standard of underwater pictures has not specifically been captured. In this paper, we tend to review the image enhancement and restoration strategies that tackle typical underwater image impairments, together with some extreme degradations and distortions. First, we tend to introduce the key causes of quality reduction in underwater pictures, in terms of the underwater image formation model (IFM). Then, we tend to review underwater restoration strategies, considering each of the IFM-free and the IFM-based approaches. Finally, we are giving an experimental-based comparative analysis of progressive IFM-free and IFM-based strategies. For that we consider the prior-based parameter estimation 
algorithms of the IFM-based strategies, mistreatment of each subjective and objective analysis. Ranging from this study, we tend to pinpoint the key shortcomings of existing strategies, drawing recommendations for future analysis during this work. Our review of underwater image enhancement and restoration provides researchers with the mandatory background to understand challenging opportunities in this vital field.

\subsection{IFM-Based Image Restoration Method}

The considering the image restoration for the removal or reduction of degradations that are enclosed throughout the acquisition of pictures e.g.; Noise, pixel value errors, out of focus blurring or camera motion blurring using prior knowledge of the degradation phenomenon. This implies it deals with the modelling of the degradation and applying the method (inverse) to reconstruct the image. The image restoration possesses a large scope of usage. The aim of image restoration is to complete or undo effects.Underwater image restoration strategies, on the opposite hand, decide to recover variety scene radiances from degraded underwater pictures. These strategies use models to investigate the mechanism of underwater image degradation, and restore the photographs by reversing the degradation method and victimisation model parameters deduced via previous data. Image restoration is completely different from image enhancement.It is meant to emphasise options of the image that build the image additional pleasing to the observer. Image enhancement techniques (like distinction stretching or de-blurring by a nearest neighbor procedure) provided by "Imaging packages" do not uses a-priori model for creating the image.

In image restoration the information provided by the microscope is only taken as indirect evidence about the image. By itself the image wants not even to be visible.A research image contains additional information than that is visible within the image.Often, details are hidden within the noise, out-of-focus signal, or masked by other features. Artifacts could confuse the viewer. Information is also given in implicit type thus it will solely be retrieved with the addition of a-priori data.

Underwater Image Color Restoration Methods:

1. Dark Channel Prior Method (DCP): Single Image Haze Removal Using DCP..

2. Green-Blue channels dehazing and Red channel Correction.: Single underwater image restoration by GB dehazing $\mathrm{R}$ correction.

3. Image Blurring and Light Absorption (IBLA): Underwater Image Restoration supported Image opaqueness and lightweight Absorption.

4. Low complexness DCP: Low complexness Underwater Image enhancement supported Dark Channel prior initial ends up in underwater single image dehazing.

5. New Optical Model: Single underwater image enhancement with a replacement optical model.

6. Ro WS: Removal of water scattering.

7. Underwater Dark Channel Prior Method(UDCP): Transmission Estimation in Underwater Single pictures.
8. Underwater Light Attenuation Prior Method(ULAP) : A fast Scene Depth Estimation Model supported Underwater lightweight Attenuation previous for Underwater Image Restoration.

\subsubsection{Maximum Intensity Projection}

Maximum Intensity Projection (MIP) consists of sticking the voxel with the best attenuation price on each read throughout the amount onto a second image. Real-time operation and visualisation of the 3D image information are foremost vital necessities for medical imaging. Among varied $3 \mathrm{D}$ visualisation strategies, most intensity projection (MIP) could be a great tool to examine 3D medical pictures. However, an oversized computation quantity could be a downside of mistreatment of the MIP image in clinical designation. The time interval of the MIP depends on the amount of voxels of the 3D information. So as to beat the massive quantity of computation for the MIP, we've developed a progressive MIP methodology which will perform the MIP with low-resolution for quick process, and use the low-resolution MIP image to get a full-resolution MIP image with a reduced computation time. By discovering the sturdy distinction in attenuation between the $\mathrm{R}$ and therefore the $\mathrm{GB}$ channels of underwater pictures, [8] Carlevaris-Bianco et al. planned a completely unique previous information for scene depth estimation and questionable most intensity projection.

The MIP methodology defined by the distinction between the most $\mathrm{R}$ channel intensity and the maximum of the $\mathrm{G}$ and $\mathrm{B}$ channels, and a shift from the highest intensity within the foreground represent a large difference between color channels. Experimental results showed that MIP may describe coarse depth maps of pictures.The MIP was conjointly used for background light(BL) estimation.The MIP to estimate the BL of underwater pictures, with the belief that the intensity of the $\mathrm{R}$ channel was comparatively under that of the GB channels within the background space.

\subsubsection{Dark Channel Prior Method}

In [7], Galdran et al. stated that the Red Channel previously supported the DCP to recover the lost distinction in underwater pictures. This new prior method reversed the red channel to modify the strong attenuation of red lightweight within the water body. In [8], Drews Junior et al. planned the Underwater DCP (UDCP) from the normal DCP by excluding the red channel in manufacturing the previous. Aside from the DCP-related priors, there are also different priors planned for underwater image restoration. In [8], Carlevaris-Bianco et al. proposed a prior by comparing the most intensity of the red channel to the most intensity within the inexperienced blue channels over a tiny low image patch. In [9] and [10], Peng et al. outlined a new prior method from image indistinctness and used it to enhance the standard of pictures from varied underwater environments. There are also other strategies that combine the options of the former 2 classes. 
For example, within the work of Hou et al. [11], the UDCP is employed beside quad-tree subdivision and Gamma correction to enhance the distinction and saturation of underwater pictures. And in [12], Qing et al. planned a comprehensive methodology with accommodative dehazing and accommodative bar graph exploit to get rid of the scattering and restore the colour balance of underwater pictures. Sometimes for these strategies, no strict classification is formed. In these strategies, the simplified image formation model (IFM) is often used for its effectiveness and ease, and because of its similarity to the model of outside hazy image, the Dark Channel previous (DCP) from outdoors image dehazing is additionally wide introduced in strategies supported this model. The DCP is accustomed to estimate the Transmission Map (TM) and scene depth. Red light possesses longer wavelength and lower frequency and thereby attenuates faster than the blue counterpart[5].

Thus the DCP supported RGB channels in underwater scenes would usually find themselves considering solely the red channel to live transmission, resulting in inaccurate depth estimation and poor restoration results. An underwater DCP support mainly the green and blue channels to avoid this drawback.

\subsection{IFM-Based Image Enhancement Method}

With image enhancement, noise can be effectively removed by sacrificing some resolution. However, this is not acceptable in many applications as no distinction can be made between noise and real signal. Image enhancement is the procedure of improving the quality and information content of original data before processing. Image enhancement widely used in computer graphics.

The principle objective of image enhancement techniques is to process an image so that the result is more suitable than the original image for a specific application. The aim of image enhancement is to improve the information in images for human viewers, or to provide 'better' input for other automated image processing techniques.

Underwater Image Enhancement Methods:

1. CLAHE: Contrast limited adaptive histogram equalization.

2. Fusion-Matlab: Enhancing underwater images and videos by fusion.

3. GC: Gamma Correction.

4. Histogram Equalization(HE): Image enhancement by histogram transformation.

5. Iterative Conditional ModesI (CM): Underwater Image Enhancement Using an Integrated Colour Model.

6. Relative Global Histogram Stretching (RGHS): Shallow-Water Image Enhancement Using RGHS Based on Adaptive Parameter Acquisition.

7. Rayleigh Distribution: Underwater image quality enhancement through composition of dual intensity images and Rayleigh stretching.

8. Unsupervised Colour Correction Method: Enhancing the low quality images using UCM.

\subsubsection{Contrast Limited Adaptive Histogram Equalization}

CLAHE[4] is a process of converting the images to grayscale and calculating the histograms of the images. The histogram equalization method is used and hence reduces the noise from the image.CLAHE is a variant of Adaptive histogram equalization (AHE) which takes care of over-amplification of the contrast. CLAHE operates on small regions in the image, called tiles, rather than the entire image. The neighboring tiles are then combined using bilinear interpolation to remove the artificial boundaries. We can also apply CLAHE to color images, where usually it is applied on the luminance channel and the results after equalizing only the luminance channel of an HSV image are much better than equalizing all the channels of the BGR image.

The method has three parameters[4]:

- Block size : The size of the local region around a pixel for which the histogram is equalized. This size should be larger than the size of features to be preserved.

- Histogram bins: The number of histogram bins used for histogram equalization. The implementation internally works with byte resolution, so values larger than 256 are not meaningful. This value also limits the quantification of the output when processing 8 bit gray or 24 bit RGB images. The number of histogram bins should be smaller than the number of pixels in a block.Use the fast but less accurate version of the filter. The fast version does not evaluate the intensity transfer function for each pixel independently but for a grid of adjacent boxes of the given block size only and interpolates for locations in between.

- Max slope: Limits the contrast stretch in the intensity transfer function. Very large values will let the histogram equalization do whatever it wants to do, that is result in maximal local contrast. The value 1 will result in the original image.

CLAHE has also two important parameters: clip limit (CL) and the number of tiles (NT).CL is a numeric value that controls the noise amplification. Once the histogram of each sub-area is calculated, they are redistributed in such a way that its height does not exceed a desired clip limit. Then, the cumulative histogram is calculated to perform the equalization. NT is an integer value which controls the amount of non-overlapping sub-areas: based on its value, the image is divided into several (usually squared) nonoverlapping regions of equal sizes. CLAHE, a histogram based image enhancement method limits amplification based on the clipping done in the histogram to a limit to a predefined level. In the proposed work, CLAHE method is used to enhance the inverse of intensity image. When CLAHE is applied in a negative image, the visual features of the image are improved and more details will be present in the enhanced image. 


\subsubsection{Rayleigh Distribution}

Rayleigh distribution is used for nonnegative-valued random variables. In the process of rayleigh, first it calculates histogram equalization then it uses color channel composition method. The average is found for the image value of saturation stretched in the image. Rayleigh Distribution is the most basic and appropriate distribution for underwater imaging. It actually refers to the bell shaped histogram distribution in which maximum pixels are concentrated at the centre of the intensity level. The pixel numbers at the minimum and maximum sides of the Rayleigh distribution are lowest to minimize the pixel amount from having too low or too high intensity values. Therefore, Rayleigh distribution reduces the pixel number of under and over contrasted areas that may be developed in the resultant image. The clip limiting process is applied to the image histogram to decrease excessive pixels for dominant intensity levels. Image histogram showing the Probability Distribution Function (PDF) and Cumulative Distribution Function (CDF) is then associated with the Rayleigh Distribution.

\subsection{Comparison of IFM-Free and IFM-Based Methods}

The Background Light (BL) estimation methodology for underwater pictures is usually neglected by researchers. It determines the colour tone and visual result of fixed pictures. Several estimation algorithms of Transmission Map (TM) additionally depend upon the results of Background Light (BL) estimation to an outsized extent.In order to match BL estimation strategies supported totally different priors, this review selects four typical pictures as well as shallow-sea fishes underneath natural source of illumination, formation underneath low-brightness scene and destroyed ships, and also the swimming spiny-finned fish within the foreground space, as exemplified.

Different bar chart techniques are examined on totally different underneath water pictures. It clearly analyzed and ascertained that distinction restricted reconciling bar chart exploit using CLAHE obtained higher results compared to reconciling bar chart exploit of AHE techniques and bar chart exploit using HE.The CLAHE improvement methodology with a clip limit of 0.2 and Rayleigh distribution method with clip limit of 0.04 will improve the standard of underwater pictures in order to optimize the results of matching underwater images.CLAHE methodology tried to optimize underwater image matching results using SURF methodology[15] (Speeded Up Robust Features) with a increase of $76.8 \%$ compared to image matching while not exploitation improvement. The SURF methodology[15] will be developed and optimized with alternative improvement strategies, to possess a high variety of matched points so the developed methodology will be enforced in an application to facilitate the identification method of underwater pictures.

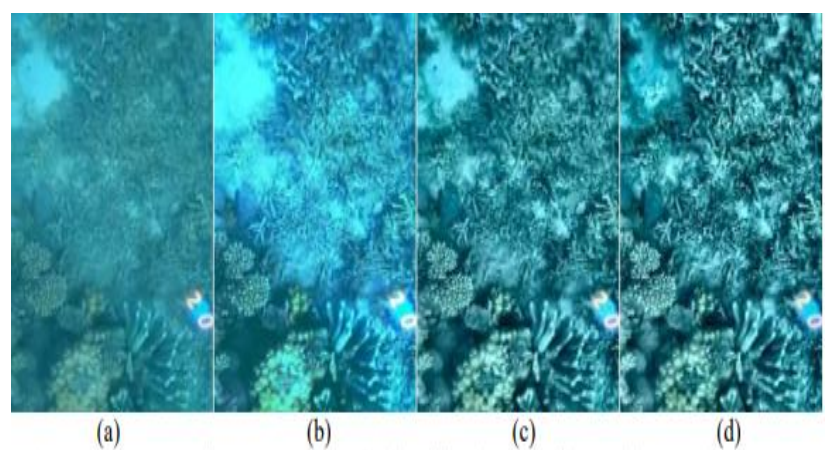

Figure 1: (a) Original Image (b) HE (c) AHE (d) CLAHE

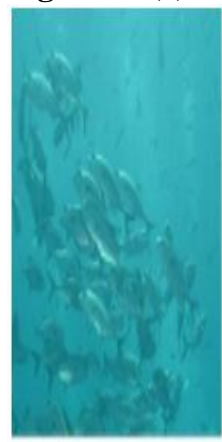

(a)

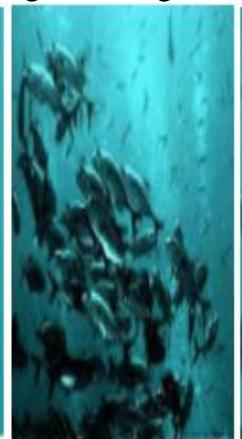

(b)

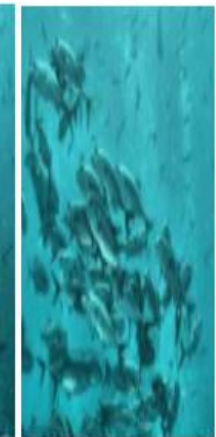

(c)

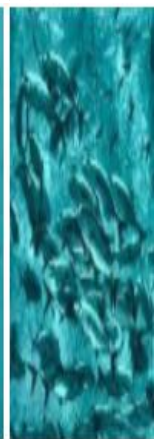

(d)

\section{Figure 2: (a) Original Image (b) HE (c) AHE (d) CLAHE}

In future, the CLAHE methodology will be improved by reducing the brightness by exploiting the Dark Channel Prior(DCP) methodology. Figure 1 and Figure 2 shows the result after getting from the HE, AHE and CLAHE Method with respect to the underwater images.

\section{RESULTS AND DISCUSSION}

Previous underwater image restoration methods used the IFM only based on the DCPs or the MIP. In this section, we compare our previous method based on image blurriness and the proposed method based on both image blurriness and light absorption against the DCP- and the MIP- based methods. The performance of the proposed method is evaluated in three ways:

1) Subjective visual comparison including examination of the depth map and the Background Light.

2) Objective quantitative full-reference assessment of restored synthesized underwater images.

3) Objective quantitative no-reference quality assessment of restored real-world underwater images.

Overall, the IFM-free methods can effectively improve contrast, visibility and luminance of the underwater image, but bring unnatural chroma and enlarged noises. The current IFM-based underwater image restoration methods can only complete basic dehazing work but cannot effectively deal with color restoration for various underwater images. But the color correction can be imported as post-processing to improve the brightness, color and contrast of restored images. According to results 
of different restoration methods, our review raises one question whether this simplified imaging formation model is actually inappropriate for underwater image restoration. The CLAHE enhancement method with a clip limit of 0.2 and a Rayleigh distribution of 0.04 can improve the quality of underwater images thus optimizing the results of matching underwater images. The below Fig 3 shows the comparison result of image enhancement and image restoration methods using CLAHE , RayLeigh Distribution, DCP and MIP. The result after getting it from these methods will vary with respect to the functionalities which are included in each method.

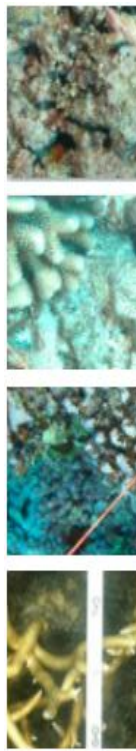

(a)
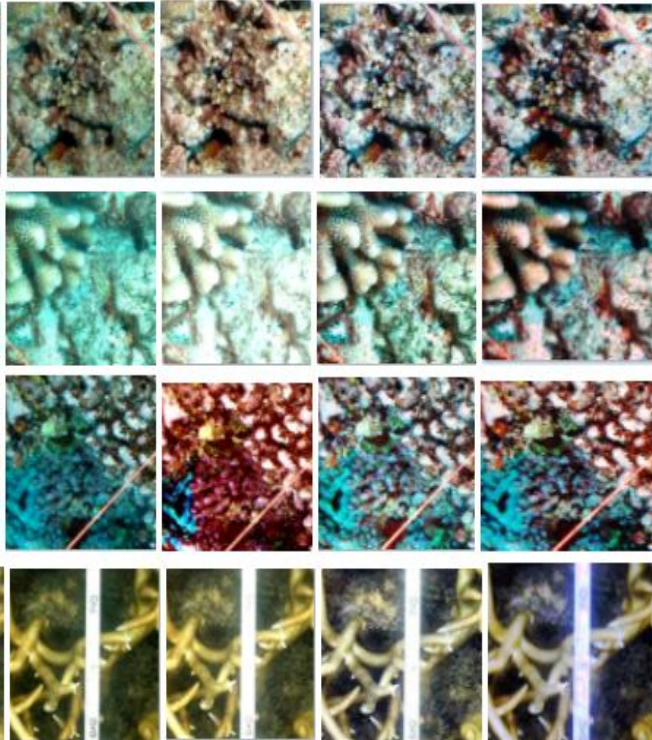

(b)

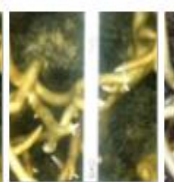

(c)

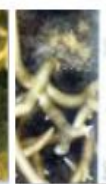

(d)

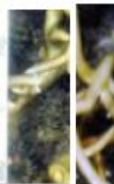

)
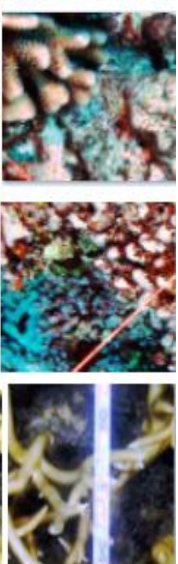

(e)
Figure 3: (a) Original Image (b) DCP (c) MIP (d) CLAHE (e) Rayleigh

Performance Evaluation Over the Four Methods we Implemented : DCP,MIP,CLAHE,RAYLEIGH

A. Mean Square Error (MSE) is calculated by the sum of square of prediction error which is real output minus predicted output and then divide by the number of data points. It gives you an absolute number on how much your predicted results deviate from the actual number.

$$
M S E=\frac{1}{N} \sum_{i=1}^{N}\left(y_{i}-\hat{y}_{i}\right)^{2}
$$

Figure 4 . Mean Square Error Formula

B. Structural Similarity Index (SSIM) measures the perceptual difference between two similar images. It cannot judge which of the two is better: that must be inferred from knowing which is the "original" and which has been subjected to additional processing such as data compression.
MSE: 722.18, SSIM: 0.91
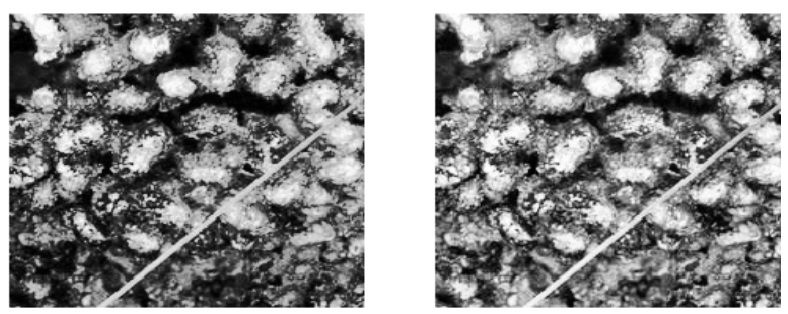

Figure 5:Rayleigh with Clahe

MSE: 593.88, SSIM: 0.91
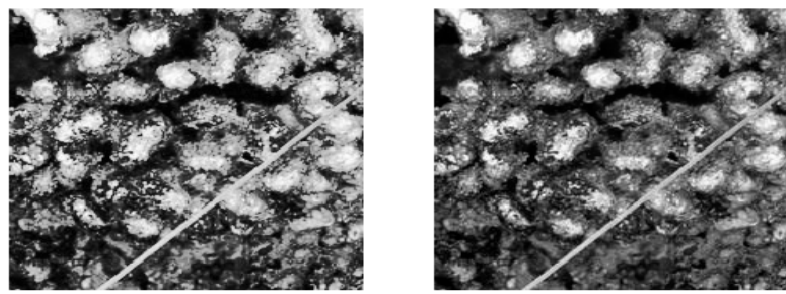

Figure 6: Rayleigh with DCP

MSE: 2512.11, SSIM: 0.66
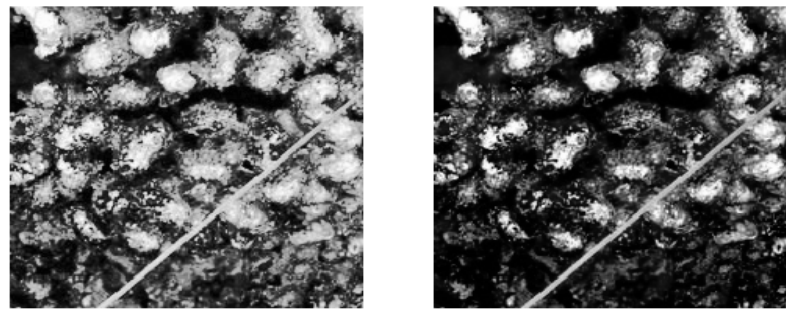

Figure 7: Rayleigh with MIP

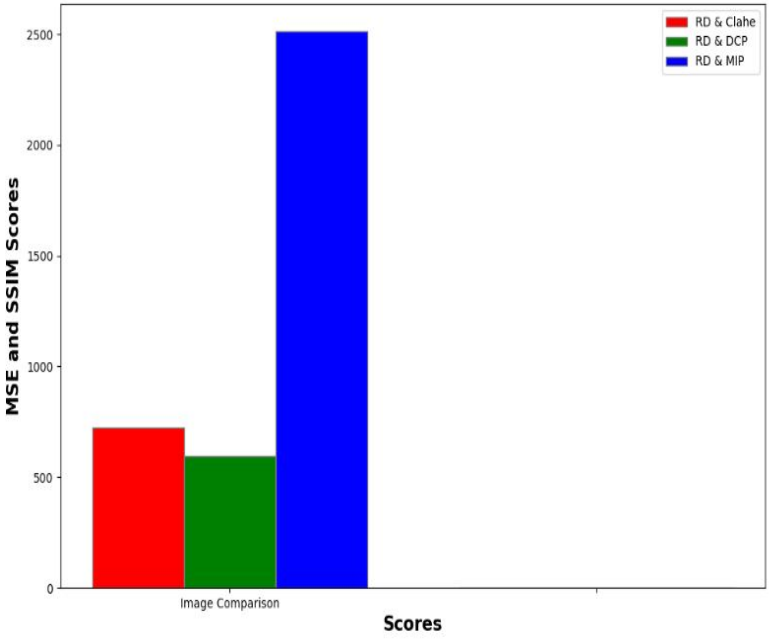

Figure 8. Graph Plotted Using MSE and SSIM 
Figure 5, Figure 6 and Figure 7 shows the compared results of methods including Rayleigh with Clahe,Rayleigh with DCP and Rayleigh with MIP which measures the performance evaluation parameters such as SSIM and MSE. The value of MSE denotes the average difference of the pixels all over the image. Here, we are measuring the difference of one method(Rayleigh) with the other three methods (DCP,MIP,CLAHE) as input for comparison. The MSE value is more in Rayleigh Distribution with MIP compared to other results, that means the difference between the images in Rayleigh and MIP is high. SSIM measures indicate that structural similarity is very low in the case of Rayleigh and DCP. By the result of this analysis, we can conclude that Rayleigh distribution method is a more effective method in the case of underwater imaging.

\section{CONCLUSIONS}

Quality improvement strategies of single underwater pictures supported image improvement and color restoration are reviewed. Summarized the standard improvement strategies of single underwater pictures into 2 classes : IFM-Free methods and IFM-Based methods. An experimental-based comparison of the progressive quality improvement strategies exploit multiple quality assessment metrics. Single underwater image improvement and restoration strategies have created tremendous progress. Nowadays there are no algorithmic programs which will be effectively applied to boost underwater pictures captured from various environments, depths or scenes.

The ability and hardness of underwater image improvement strategies still ought to be improved. Additionally, ancient enhancement/restoration algorithms have comparatively high quality with considerable limitations to our ability to rescale sensible studies and applications. Different bar chart techniques are examined on totally different underneath water pictures. It was clearly analyzed and determined that distinction restricted reconciling Contrast limited adaptive histogram equalization (CLAHE) obtained better results compared to Adaptive Histogram Equalization (AHE) techniques and Histogram equalization (HE). Based on this analysis, We can summarize the Rayleigh Distribution as a more effective method for underwater imaging if we compare with other results such as Clahe,DCP and MIP.

\section{ACKNOWLEDGEMENT}

We would like to thank the anonymous reviewers for their insightful comments that helped greatly improve the paper. We also acknowledge support for this project from the department of Computer Science and Information System at Rajagiri School Of Engineering and Technology.

\section{REFERENCES}

1. Underwater image processing system for image enhancement and restoration, chengyi cai, yiheng zhang, ting liu ,2019.

2. An underwater image enhancement algorithm for environment recognition and robot navigation, kun xie, wei pan and suxia 361005,2017.

3. Enhancement of Underwater Images With Statistical Model of Background Light and Optimization of Transmission Map, Wei Song, Member, IEEE, Yan Wang, Dongmei Huang, Antonio Liotta, Senior Member, IEEE, and Cristian Perra, Senior Member, IEEE,2020.

4. A survey of restoration and enhancement for underwater images, weidong zhang 1 , lili dong 1 , 2019.

5. Underwater image enhancement by wavelength compensation and dehazing, weidong zhang 1 , lili dong 1, 2019.

6. Underwater image restoration using color correction and non-local prior, meng wu, member, IEEE, kai luo, jianjun dang, and daijin li, 2017.

7. Galdran, A.; Pardo, D.; Picón, A.; Gila, A.A. Automatic Red-Channel Underwater Image Restoration. J. Visual Commun. Image Represent. 2015, 26, 132-145

8. Drews, P., Jr.; Nascimento, E.; Moraes, F.; Botelho, S.; Campos, M. Transmission Estimation in Underwater Single Images. In Proceedings of the The IEEE International Conference on Computer Vision (ICCV) Workshops, Sydney, Australia, 1-8 December 2013.

9. Carlevaris-Bianco, N.; Mohan, A.; Eustice, R.M. Initial Results in Underwater Single Image Dehazing. In Proceedings of the OCEANS 2010 MTS/IEEE SEATTLE, Seattle, WA, USA, 20-23 September 2010.

10. Peng, Y.T.; Zhao, X.Y.; Cosman, P.C. Single Underwater Image Enhancement Using Depth Estimation Based on Blurriness. In Proceedings of the 2015 IEEE International Conference on Image Processing (ICIP), Quebec City, QC, Canada, 2730 September 2015.

11. Peng, Y.T.; Zhao, X.Y.; Cosman, P.C. Underwater Image Restoration Based on Image Blurriness and Light Absorption. IEEE Trans. Image Process. 2017, 26, 1579-1594.

12. Hou, G.J.; Pan, Z.K.; Wang, G.D.; Yang, H.; Duan, J.M. An Efficient Nonlocal Variational Method with Application to Underwater Image Restoration. Neurocomputing 2019, 369, 106-121.

13. Abdul Ghani, A.S., Mat Isa, N.A., 2015. Enhancement of low quality underwater image through integrated global and local contrast correction. Appl. Soft Comput. 37, 332-344. [2] Li, Y., Lu, H., Li. J., Li, X., Serikawa, S., 2015.

14. Underwater image enhancement using inherent optical properties. In: Proceeding of the 2015 IEEE, International Conference on Information and Automation. Lijiang, China. August 2015. pp. 419422.

http://dx.doi.org/10.1109/ICInfA.2015.7279324

15. An Analysis of the SURF Method,ENS, D'epartement Informatique, France,Image Processing On Line 2015,Edouard Oyallon, Julien Rabin. 